incomplete work be checked, but also the number of papers published would be halved, for the discussion would show the writer that half the facts he would normally record are insignificant details having no bearing on his general argument.

Secondly, the suggestion of Drs. Berry and Bonser that there is a tendency for the young research worker to be judged upon the number of his papers rather than on their quality, gives rise to many thoughts which cannot be touched on here. But it does prompt the question as to how much research is done with the idea of the advancement of some branch of science and how much for added professional qualification. To-day, even for minor posts, candidates are expected to have research qualifications; and quantity rather than quality will continue to count unless those responsible for making such appointments are prepared either to seek such expert advice on the publications of the candidates as they would for a senior post, or to pay less attention to this aspect of the qualifications.

Whether the apparent respect paid to all forms of research is genuine is another matter, for the reward for the most brilliant research would appear to be, not the means and leisure to continue it, but a post involving such administrative or teaching duties as to make further research impossible.

Department of Zoology,

University of Glasgow.

${ }^{1}$ Nature, 135, 664, April 27, 1935.

\section{Plasticity of Rock Salt and the Taylor and Becker- Orowan Theories of Crystalline Plasticity}

IN Prof. Taylor's theory of crystalline plasticity ${ }^{1}$ the process of slip is. conceived as the result of the propagation through the lattice of a definite type of deviations from the 'ideal' structure, so-called dislocations ${ }^{2}$. The path of a single dislocation in general is limited by the faults or flaws in the crystal and it is assumed that the number $N$ of propagated dislocations increases during the course of the process. Every dislocation is the centre of a field of stress; the various dislocations influence each other's motions and it is shown that centres will escape from each other only if the applied exterior stress exceeds a certain value, increasing with $N$. Hence the stress necessary for further deformation increases with the deformation already attained ('shear hardening'). It is suggested by Taylor that the dislocations might arise as a consequence of thermal agitation, but no explicit explanation is given of the progressive increase of $N$.

Now, in the Becker-Orowan theory, gliding is conceived as starting from 'jumps', originating at favourably situated flaws under the combined influence of the exterior stress $\tau$ (enhanced by the socalled stress-concentration effect), and of thermal agitation (producing local stress fluctuations ${ }^{3}$ ). This conception leads to a relation between $\tau$ and the rate of flow, from which also the minimum value $\tau_{f}$ necessary to produce an observable rate of shear at a given temperature can be estimated. It is put forward by Orowan (l.c., p. 639) that these local jumps initiate dislocations of similar type as considered in Taylor's theory. If such dislocations, once formed, move only over a finite distance and are then arrested at 'opaque' flaws, it would seem to us that they will give rise to an 'interior' field (similar to that introduced by Taylor, though now essentially connected with the lattice distortions at the places where dislocations have been arrested), of a stress $\tau_{i}$ increasing with the number of dislocations formed. In those regions where jumps have occurred the interior field in the main will counteract the exterior field, and thus we must expect that the production of new dislocations will take place at a continuously decreasing rate. When $\tau_{i}$ has increased so much that $\tau-\tau_{i}$ approaches to the value $\tau_{f}$, the deformation practically comes to a stop, as then new dislocations are formed so slowly that their only effect will be a very small creep. Reasoning along these lines it seems possible to arrive at a combination of the two theories, which explains both the shear hardening effect and the dependence of the rate of flow upon exterior stress and temperature ${ }^{4}$.

The picture obtained suggests that the plastic properties of a crystal will be enhanced if it is possible to keep down the interior field $\tau_{i}$. It seems worth while to consider from this point of view the remarkable fact, observed by Smekal ${ }^{5}$, that on stretching a piece of rock salt the actual gliding among several equivalent sets of glide planes chooses that set, for which the direction of gliding occupies the shortest way in the piece. If it is assumed that a certain proportion of the dislocations which arrive at the surface layer of the crystal are able to 'escape', so that they do not contribute to the interior field, the interior field for a given total shear will be the smaller when the number of dislocations that can arrive at the surface is greatest, which will be the case for the shortest glide path.

To some extent, similar reasoning may perhaps help to understand the much discussed influence of water on the plasticity of rock salt. If the water has a 'healing' effect on the surface layers, those dislocations which were arrested by flaws in these layers can escape, and thus a decrease of $\tau_{i}$ will be effected. This means that a definite exterior stress can give rise to the birth of a larger number of dislocations and thus to a larger shear.

The above suggested 'explanation' of the Joffé effect as a 'surface effect' apparently fits in with the conceptions of W. Ewald and M. Polanyi ${ }^{6}$. If it were assumed, as has been proposed by Smekal and co-workers ${ }^{7}$, that the water penetrates into the crystal, an increase in plasticity could be effected also if the 'opacity' of internal flaws for the passage of dislocations were diminished, thus increasing their mean 'free path' $L$. This second explanation would be similar in nature to that accepted by Taylor for the influence of temperature on the degree of plasticity.

$$
\text { W. G. Burgers. }
$$

Natuurkundig Laboratorium

der N. V. Philips' Gloeilampenfabrieken, Eindhoven, Holland.

J. M. Burgers.

Aerodynamical Laboratory, Delft. March 18.

1 G. I. Taylor, Proc. Roy. Soc., A, 145, 362, 388, 405; 1934.

2 Compare also M. Polanyi, Z. Physik, 89, 660; 1934.

${ }^{3}$ R. Becker, Physik. Z., 26, 919 ; 1925. E. Orowan, Z. Physik, 89, $605,614,634 ; 1934$

$89,605,614,634 ; 1934$. "This will be set forth in more detail in Chapter V (section 11) of a 15, No 3 .

15, No. 3; 1935). Physik, 98, 166; 1935.

W. Swald and M. Polanyi, Z. Physik, 28, $29 ;$ 1924. M. Polanyi, ibid., 89,660; 1934

7 See, for example, K. Wendenburg, ibid., 88, 727 ; 1934. 